\title{
SPIN TRANSPORT IN SEMICONDUCTOR HETEROSTRUCTURES
}

Final Report

D. C. Marinescu

Department of Physics and Astronomy, 207 Kinard Laboratory. Clemson University Clemson, SC 29634-0978;

Ph: (864)656-5315;

Fax: (864)656-0805;

Email:dcm@clemson.edu

\section{Prepared for}

THE U. S. DEPARTMENT OF ENERGY AWARD NO. DE-FG02-01ER45897 


\section{NOTICE}

This report was prepared as an account of work sponsored by the United States Government. Neither the United States nor the United States Department of Energy, nor any of their employees, makes any warranty, express or implied, or assumes any legal liability or responsibility for the eccuracy, completeness, or usefulness of any information, apparatus, product or process diaclosed of represents that its use would not infringe privately-owned rights. 


\section{Contents}

1 Description of Accomplikhments 4

1.1 Introduction . . . . . . . . . . . . . . . . . . . . . . . . 4

1.2 Collective excitations in bi-layer spin-polarized systems . . . . . . . 4

1.3 Effective electron-electron interaction in spin polarized systems . . . . . . . 5

1.4 Pair Distribution Function in A Two Dimensional Electron System . . . . 6

1.5 Dynamic Coupling in Spin Polarized Transport; The Spin Mass of an Electron Liquid .......................... . . . 7

1.6 Spin Trarsresistivity - A Probe of the Electron-Electron Correlations . . . 8

1.7 Spin Dynamics in the presence of the Spin-Orbit Coupling . . . . . . . . 9

2 Publication List: July 2001-September 2005 11

2.1 Papers Published or Accepted by Refereed Journals . . . . . . . . . . . . . . 11

2.2 Manuscripts in Preparation . . . . . . . . . . . . . . . . 11

2.3 Research Ascociates Progress Report . . . . . . . . . . . . . . . . . 12

3 Collaboration with the Oak Ridge National Laboratory 12 


\section{Description of Accomplishments}

\subsection{Introduction}

The focus of the research performed under this grant has been the investigation of spin transport in magnetic semiconductor heterostructures. The interest in these systems is motivated both by their intriguing physical propertim, as the physical embodiment of a spin-polarized Fermi liquid, as well as by their potential applications as spintronics devices. In our work we have analyzed several different problems that affect the spin dynamics in single and bj-layer spin-polarized two-dimensional (2D) systems. The topics of interests ranged from the fundamental aspects of the electron-electron interactions, to collective spin and charge density excitations and spin transport in the presence of the spin-orbit coupling. The common denominator of these subjects is the impact at the macroscopic scale of the spin-dependent electron-electron interaction, which plays a much more subtle role than in unpolarized electron systems. Our calculations of several mensurable parameters, such as the excitation frequencies of magneto-plasma modes, the spin mass, and the spin transresistivity, propose realistic theoretical estimates of the opposite-spin many-body effects, in particular opposite-spin correlations, that can be directly connected with experimental messurements.

The results of this effort bave been published in major physics journals and helped establish our group as an active center of spintronics research. The research associates affiliated with this project went on to independent research positjon, an unofficial but important recognition of the quality of our work. During the past four years our traditional collaboration with Dr. Randy Fishman, from the Condensed Matter Division of the Oak Ridge National Laboratory has strengthened, while new collaborative venues with other important groups in spintronics, from the University of Missouri and Scuola. Normale in Pisen Italy, have been opened. On a personal level, this grant gave me the opportunity to improve and refine my

leadersbip skills, allowed me to advance professionally by getting tenure and being promoted to associate professor, and be recognized as an important contributor to the general spintronics effort by my peers who invited me to participate to the spintronics symposium organized next spring at the Kavli Institute in Santa Barbara.

Below, I will discuss our results, presented in chronological order.

\subsection{Collective excitations in bi-layer spin-polarized systems}

In the first part of the project, Dr. Ionel Tifrea and I investigated the collective excitations triggered by an external electromagnetic field in a bilayer system - two similar quantum wells placed side by side, between which no tunneling is permitted. The importance of this subject stems from its connection to the effective electron-election interaction that determines the difference between the excitation frequencies of the collective modes and single particle excitations. Moreover, any theoretical predictions of these values can be readily verified by infrared spectroscopy experiments, and thus provide an early inkling on the correctness of the microscopic model chosen to describe the interection.

In our paper, "Magneto-Acoustic PIasmons in a Bilayer Quasi Two Dimengional Spin Polarized System" [Phys. Rev, B 64, 073405 (2001)], we calculate the charge and spin response functions of a bilayer quasi two dimensional (2D) system, spin polarized by a constant 
magnetic field $\vec{B}$. Terme beyond the random-phase approximation (RPA), the exchange (x) and correlation (c) interactions, are introduced by using generalized spin-dependent local field factors, $G_{\sigma}^{\mathrm{r}, c}(\vec{q}, \omega)$. The self-consistent magnetic interaction among the electron spins determines the coupling of the charge and the longitudinal spin density excitations, leading to coupled in-phase and out-of-phase electric and magnetic modes. We find that the lowest. frequency belongs to an acoustic mode, that represents the out-of-phase oscillation of the longitudinal magnetizations in the two layers. This collective excitation is shown to become important in the case of materials with large gyromagnetic factors, such as dilute magnetic semiconductors. The experimental verification of this collective excitation is expected to give important information on the microscopic nature of the self-consistent magnetization in quasi-two dimensional sygtems.

In an immediatc follow-up, we analyzed the spin and charge coupling of both intra- and inter-band excitations in "Collective Modes of a Bilayer Quasi-Two-Dinensional Spin Polarized Electron Gas" [Physica E 15, 13 (2002)]. In this paper we show that, in the presence of a weak electromagnetic perturbation, a biloyer quasi-two-dimensional spin polarized system exhibits coupled in-phase and out-of-phase charge and spin density resonant excitations. These modes occur when either the self-consistent magnetization or Coulomb interaction effects beyond the random phase approximation (RPA) are considered. We analyze both intraand inter-subband excitations and provide analytic results for their excitation frequencies in the long wavelength limit.

The more challenging gituation, when the two layers are coupled through quantum tunneling, is addressed in "Collective Modes in Coupled Quasi-Two-Dimensional Spin-Polerized Layers" [Phys. Rev, B 65, 113201 (2002)]. Here, we investigate the occurrence of charge and spin density collective excitations in a bilayer quasi-two-dimensional spin polarized system in the presence of interlayer tunneling. The inclusion of the self-consistent magnetization interaction among the electron spins, in addition to the Coulomb interaction, permits the resolution of coupled in-phase and out-of-phase electric and magnetic modes associated with the charge and spin densities in the two layers. While the in-phase modes are not affected by tunneling, the out-of-phase modes, both electric and magnetic, are gaped, the width of the gap being proportiongl to the tunneling anplitude. We analyze the existence of these modes outside the electron-hole continuum within the random phase approximation and suggest that they can be relevant for materials with large gromagnetic factors, for example, dilute magnetic semiconductors.

\subsection{Effective electron-electron interaction in spin polarized sys- tems}

Obtaining the exact frequency and wave vector dependence of the Iocal field corrections is a very difficult problem that has remained, as yet, unsolved even in the case of the unpolarized electron system. Fortunately, their asymptotic values can be obtained exactly in two limiting casee. At zero frequency and smatl wavevectors sum rules are used to connect the static limits of the response functions to centain thermodynamic coefficients. For large frequency and large wavevector, an iterative method generates the exact expressions for the local field functions to second order. This approach involves the equation of motion satisfied by the 
Wigner distribution function of the particle density.

Building upon an earlier work, where the zero frequency and long wavelength limits of $G_{v}^{x, c}(\omega, \vec{q})$ were determined, [D. C. Marinescu and J. J. Quinn, Phys. Rev. B 56, 1114 (1997)], a second step in understanding the dynamic electron-electron spin-dependent interaction was taken in "Thermodynamic Limit of the Locall Field Correction Factors in Spin Polarized Electron Systems" [Phys. Rev. B 65, 113201 (2002)]. In this work we obtain the zero frequency and long wavelength limits of $G_{\sigma}^{x, c}(\omega, \vec{q})$ by analyzing the connection between the macroscopic response function and the thermodynamic parameters of the system.

Numerical estimates of the response functions of the three dimensional unpolarized electron gas have shown that local field factors smoothly interpolate between the asymptotic smill and large wave-vector behavior. This feature is expected to exist also in the case of a spin polarized system and consequently, we use the exact asymptotic values of $G_{v}^{x_{1} c}(\mathbf{q}, \omega)$ for large and small momentum at zero frequency as a starting point in obtaining their epproximate expressions across the whole spectrum of momentum. Dr. Juana Moreno and I obtained the spectral behavior of the local field corrections in our paper entitled "Local field factors in a polarized two-dimensional electron gas", [Phys. Rev. B. 68,195210 (2003)]. In this work we derive approximate expressions for the static local field factors of a spin polarized two-dimensionsl electron gas, that in the absence of spin polarization reproduce recent diffusion Monte Carlo data. We find that the degree of spin polarization produces important modifications to the local factors of the minority spins, while the local field functions of the majority spins are less affected. As a continuation, we will analyze the effective electron-electron interaction in the spin-polarized system.

\subsection{Pair Distribution Function in A Two Dimensional Electron System}

The large wavevector aud the high frequency limits of the local field factors depend essentially on the zero inter-electronic distance value, $g(r=0)$, a quantity also relevant in non-local density functional theories. The importance of $g(r)$ lies in its connection with the electronic exchange and correlation of the electron gas model. Moreover, theoretical calculations of the pair diatribution function can be directly compared with material properties since $g(r)$ is the Fourier transform of the static structure factor.

The pair-distribution function is the probability of finding a pair of electrons at a distance $r$ from each other. Therefore, the average number of electrons in a spherical shell centered on an given electron is $n g(r) \Omega_{D} r^{D-1} d r$, where $\Omega_{D} r^{D-1} d r$ is the volume of the D-dimensions] shell and $n=N / V$ is the uniform electron density. At large distances, $g(r)$ approaches 1, whereas near the origin, where the electron charge is depleted, it is small on account of the Pauli exclusion principie and the exchange and correlation effects associated with the Coulomb interaction.

In our paper "Pair distribution function at the origin in a two-dimensional electron gas" [J. Phys.: Condens, Matter 15, 6321-6329 (2003)] we investigate the pair correlation function dependence on the inter-electronic distance and electron density of in a two dimensional, interacting, spin polarized electron system. To this purpose, we follow the approach developed in three dimensional systems by Overhauser [A. W. Overhauser, Phys. Rev. B 3, 1888 
(1971)]. This method is based on the relation between $g(r)$ and the two-electron scattering problem in an appropriatcly chosen effective potential which will be discussed in detail in the following sections. In addition to obtaining the variation of the pair distribution function as a function of the coupling-strength, $T_{s}$, and of the spin polarization, we also derive an analytic expression for $g(0): g(0)=\frac{1}{2} \sqrt{1+0.6032 r_{*}+0.07263 r_{s}^{2} t^{2}}$. This expression is found to agree very well with the results of the most recent numerical calculations [C. Bulutay and B. Tanatar, Phys. Rev. B 65, 195116 (2002)] indicating a better agrement than the most recent calculation in M. Polini, G. Sica, B. Davoudi and M. P. Tosi, J. Phys.: Condens. Matter 13, 3591 (2001).

\subsection{Dynamic Coupling in Spin Polarized Transport; The Spin Mass of an Electron Liquid}

In the simplest description, that neglects all electron-electron interactions, spin transport involves the independent, simultaneous motion of opposite spin electrons driven by an effective electric field. The direction of the spin, as up, $\sigma$ or down, $\vec{\sigma}$, is assumed to be fixed either by an external magnetic field or by some injection process. The long spin relaxation times ( $\tau \sim 1 \mathrm{~ns}$ in metals and $\tau \sim 10 \mathrm{~ns}$ in semiconductors) allow a meaningful description of the transport process in terms of spin dependent transport equations which are controlled by scattering times of the order of $10^{-3}-10^{-4}$ ns. This independent spin channel picture has been used to arblyze the spin diffusion in semiconductors and to predict various possible applications to spintronic devices.

The incorporation of the electron-electron interaction introduces a coupling between the spin channels, described in generad terms as the apparition of a spin $\bar{\sigma}$ current in the presence of a spin $\sigma$ curtent. One such occurtence is the spin drag effect, where the coupling in realized by the momentum exchanged between electrons of opposite spins through inelastic Coulomb collisions, the net result being the slowing down of the fastest spin current. When the driving force of the current is removed, the two spin currents are equal and of opposite polarization and the cffect distappears.

In the paper entitled "Dynamic Spin Coupling in Spin Polarized Transport" [J. Phys.; Condens. Matter 15, 3759-3765( 2003)], I point out a different source of dynamic spin coupling. In a phenomenological description, this effect is generated by the change in the local energy of a quasiparticle (QP) of spin $\sigma$ when both $\sigma$ and $\bar{\sigma}$ distribution functions are driven out of equilibrium by a spin dependent perturbation. Under these circumstances, the spin $\sigma$ current implied by the continuity equation is different from the same spin current associated with the momentum transport. This difference is proportional to the relative drift velocity of the two spin species:

$$
\vec{j}_{d}=-\alpha\left(\vec{v}_{a}-\vec{v}_{a}\right)
$$

where $\alpha$ is determined by the angular average of the opposite spin interaction. Such an effect is a result of the opposite spin correlations and consequently, its presence is supported only in an approximation of the many body interaction that considers explicitly the exchange and correletion effects betwen quasiparticles. From a different perspective, this is equivalent, with a renormalization of the ordinary effective mass that is different than the one in charge transport. 
This problem was developed in collaboration with Prof. Giovanni Vignale from U. of Missouri, and resulted in a PRL publicetion entitled appropriately "The Spin Mass of an

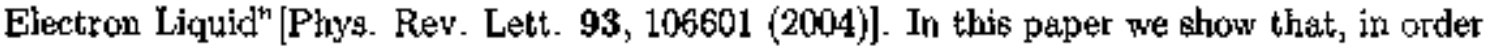
to calculate the spin current correctly, one must recoguize that the effective spin mass $m_{s}$, which determines the relation between the spin current and the quasiparticle momentum, is neither the band mase $m_{b}$ (which controlls the charge current), nor the quasiparticle mass $m^{*}$ (which controls the heat capecity), but rather a new many-body quantity, controlled by spin correlations. Our calculations show that the spin mass, in spite of uncertainties due to the approximate character of the many-body theory, can be considerably larger than the bare band mass in a two-dimensional electron gas (by contrast. the quasiparticle effective mass is typically very close to the band mass). Hence, the spin mass will have to be taken into account whenever a quantitative comparion between theory and experiment is desired.

We start from the analysis of the Boltzmann transport equation in a spin-polarized system and show that in order to satisfy the continuity equation for the electric charge, one reeds to introduce a modified effective mass, the spin mass. This is the effective mass of a quasiparticle of spin $\sigma$ and momentum $\vec{p}$ that participates in the spin current. The physical phenomenon behind this result is the momentum transferred from the up- to the down-spin component of the liquid: one may say that the up-spin qussiparticle drags along some downspin electrons es part of jts "screening cloud". We connect this momentum transfer with the angular average of the antisymmetric quasiparticle interaction.

\subsection{Spin Transresistivity - A Probe of the Electron-Electron Cor relations}

It has been recognized a long time ago that the many-body interaction in an itinerant electron system acquires a magnetic component when the short range effects, like exchange ( $x$ ) and correlations (c), are explicitly included through corresponding local field corrections $G^{x}{ }^{x}$.

Characteristically, their reflection on the macroscopic properties of the spin unpolarized system is done through linear combinations, denoted $G^{+}=G_{\sigma}^{x}+G_{\sigma a}^{c}+G_{a d}^{c}$ for the spin symmetric quantities, such as the dielectric constant, effective mass, or plasma oscillations, and $G^{-}=G_{\sigma}^{x}+G_{o s}^{c}+G_{o s}^{c}$ for spin antisymmetric quantities, as the magnetic susceptibility and spin waves. Since the same spin electrons are kept apast on account of the Pauli exclusion principle, the opposite spin correlation factor is dorninant. Among the local feld factors introduced above, the exchange one can be calculated exactly, while the correlation one is based on some approximation of the many body interaction. The experimental measurement of the various macroscopic quantities can serve as velidation for the theoretical approximations of the many-body interaction used in estimating the local field factors.

In the paper entitled "Spin Transtesistivity - A Probe of the Electron-Electon Correlations" [Solid State Commun. 129/10,649-653 (2003)] we discuss many-body corrections to the spin transresistivity, a macroscopic parameter determined by the dynamic momentum exchange between opposite spin electrons in spin polarized transport. Our results show that the first order correction beyond the RPA value is determined by $\mathrm{G}^{+}-G^{-}$, a difference proportional to the local field factor that describes correlations between opposite spin electrons. This represents a unique experimental opportunity to examine independently the opposite 
spin correlation contribution to the many-body interaction. Any deviation from the RPA value detected in a measurement of spin transresistivity should reflect this contribution.

The micrascopic origin of the spin transresistivity is the spin dependent, dynamic manybody effects, and its first order correction beyond RPA will carry over the signature of the spin dependent component of the electron-electron interaction. In order to pursue this analysis, we first derive the general expression of the spin transresistivity in a transport theory approach and then employ a spin-dependent effective interaction to estimate the scattering matrix that determimes its magnitude. We find that the first order many-body correction to the spin transresistivity is determined by the opposite spin correlation local field factor.

This work was done in collaboration with Professor Mario Tosi from Scuola Normale Superiore, Pisa and the International Center for Theoretical Physics, Trieste, Italy.

\subsection{Spin Dynamics in the Presence of the Spin-Orbit Coupling}

Known to exigt for a long time, the spin-orbit coupling in two dimensional electronic sygtems (2DEG) has received a lot of attention lately motivated by its potential applications in spintronics. Recent experiments have demonstrated that the magnitude of the spin-orbit coupling can be modified by a woltage gate, hence generating the premise of the possible manipulation of spin currents by electric fields alone. The two sources of the spin-orbit coupling are the inversion asymmetry of the confining potential in the direction perpendicular to the 2DEG (Rashbe) and the bulk asymmetry and interface inversion asymmetry (Dresselhaus).

In a very interesting development, Sinova et. al ptedicted that, a Rashba interaction induces a spin-Hall current of the transverse spin component as a response to a in-plane electric field, with a universal value $e / 8 \pi$. This intrinsic spin-Hall effect is quite different from the extrinsic spin-Hall effect proposed by Hirsch, which is generated by impurity scattering.

The possible existence and persistence in disordered systems of the intrinsic spin-Hall effect (SHE) has received a great deal of attention from the research community. The question of whether arbitrary small amounts of disorder suppress or not the intrinsic SHE is still awaiting a definite answer. Some artalytical calculations claim that SHE does not survive even in the weak disorder regime, while others provide arguments that $\mathrm{SHE}$ is robust and weak disorder in the system is not errough to destroy this effect. While the problem was studied in more detailed using analytical methods, there are few unbiased numerical calculations at present.

In our paper, entitled "Longitudinal and spin-Hall conductance of a two-dimensional Rashba system with arbitrary disorder" [Phys. Rev. B 72, 165335 (2005)], we calculate the longitudinal and spin-Hial conductances in four-probe bridges with Rashba - Dresselhaus spin-orbit interactions and present numerical results that angue for the robustness of the spin-Hall effect in the presence of disorder. We study the combined effect of the Rashba and Dresselhaus spin-orbit coupling, as well as the effect of disorder on the longitudinal and spinHall conductances by using the Landauer-Buttiker formalism (LB) combined with a Green function approach. The numerical results are obtained both within the Landauer-Büttiker formalism and by direct evaluation of the Kubo formula for clean and disordered systems. The microscopic Hamiltonian is described in tems of the neareast-neighbor hopping integra] t. Rashba spin-orbit coupling $V_{R}$, Dresșelhaus spin-orbit coupling $V_{D}$ and an Anderson-Iike, 
on-site disorder energy strength $W$. We reconfirm that below a critical disorder threshold, the spin-Hall effect is present. The similarity of our results obtained within the two different formalisms is a further argument that that intrinsec spin-Hall effect truly is a bulk property of the system.

In another paper, entitled "Longitudinal and spin-Hal conductance of a one-dimensional Aharonow-Bohm ring" [accepted for publication in $\mathrm{J}$, of Phys. Condens. Matt we formulate the one-dimensional version of this problem, for an electron gas with spin-orbit interaction confined to a quasi one-dimersional Aharonov-Bohm ring. The longitudinal and spin-Hall conductivities are studied as a function of disorder, magnetic flux and system size. The interplay of the Rashba and Dresselhaus terms of the spin-orbit interaction is discussed. As before, our results are derived within a spin-dependent Landauer-Buttiker formalism implemented with interacting electron Green's functions. We reconfirm the robustness of the intriosic spin-Hall effect against disorder and study the dependence of its amplitude on the Rashba and Dresselhane interaction, 


\section{Publication List: July 2001-September 2005}

\subsection{Papers Published or Accepted by Refereed Journals}

1. I. Tifrea and D. C. Marinescu, "Magneto-acoustic Plasmons in a Bilayer Quasi Two Dimensional Spin Polarized System ${ }^{n}$; Phys. Rev. B 64, 073405 (2001).

2. I. Tifrea and D. C. Marinescu, "Collective Modes in Coupled Quasi-Two-Dimensional Spin- Polarized Layers", Phys. Rev. B 65, 125316 (2002).

3. 1. Tifrea and D. C. Marinescu, "Collective Modes of a Bilayer Quasi-Two-Dimensional Spin- Polarized Electron Gas", Physica E 15, 13 (2002).

4. D. C. Marinescu and I. Tifrea, "Thermodynamic Limit of the Local Field Correction Factors in Spin Polarized Electron Systems", Phys. Rev. B 65, 113201 (2002).

5. D. C. Marinescu, "Spin Backflow in Spin Polarized Transport", J. Phys.: Condens. Matter 15, 3759-3765( 2003).

6. Juana Moreno and D. C. Marinescu, "Pair Distribution Function in a 2D Electron Gas", J. Phyz.: Condens. Matter 15, 6321-6329 ( 2003).

7. Juana Moreno and D. C. Marinescu, "Local Field Corrections in A Two Dimensional Spin Polarized Electron System", Phys. Rev. B. 68,195210 (2003).

8. D. C. Marinescu and M. P. Tosi, "Spin Transresistivity - A Probe for the Opposite Spin Correlations", Solid State Commun. 129/10, 649-653 (2003).

9. Juana Moreno and D. C. Marinescu, "Hartree-Fock Energy of a Density Wave in a Spin Polarized Two-Dimensional Electron Gas", J. Phys.: Condens. Matter 16, 2311 (2004).

10. Zhixin Qian, Giovanni Vignale and D. C. Marinescu, "The Spin Mass of an Electron Liquid", Phys. Rev. Lett. 93, 106601 (2004).

11. C.P. Moca and D. C. Marineseu, "Longitudinal and Spin-Hall Conductance of a TwoDimensional Rashba System With Arbitrary Disorder", Phys. Rev. B 72, 165335 $(2005)$.

12. C. P. Moca and D. C. Marinescu, "Longitudinal and spin-Hall conductance of a onedimensional Aharonow-Bohm ring", accepted for publjcation in J. Phys.: Condens. Matter.

\subsection{Manuscripts in Preparation}

1. Juana Moreno and D. Q. Marinescu "EFective electron-electron interaction in a spin polarized electron system", to be submitted to Phys. Rev. B. 
2. C.P. Moca, D.C. Marinescu, E.V. Macocian and S.M. Filip, "Spin-Hall conductance of a one dimensional Aharonov-Bohm ring with Hubbard interaction", to be submitted to Phys. Rev. B

\subsection{Research Associates Progress Report}

Over the period of this grant, three resegrch associates have been involved in this project. They are Ionel Tifrea, Juana Moreno, and C. Pascu Moca.

Ionel Tifrea came to Clemson in 2000 as a postdoctoral reaearch associate and from the beginning, our collaboration was focused on issues related to the subject of this grant, prirnarily spin and charge collective excitations in multilayer systems. Even though he was not supported by this grant, much of our activity occurred while this grant proposel was under review and his contribution in solving sone of the problems directly related to this awad is important. In the fall of 2001 , he accepted a reeearch associate position with the group of Michael Flatte at the Eniversity of Lowa. In September 2005, he returned to his Alrna Mater, Babes-Bolyai University in Cluj, Romania, where is ans Associate Profersor of Physics.

Juana Moreno joined my group at Clemson in October 2001, following a PhD at Rutgers University, and postdoctoral appointments at the Institute of Theoretical Physics in Triegte, Italy, and Nortbwester University. Our research concerned the spin dependence of the short range Coulonb interaction as it is reflected in the local field corrections. Taking advantage of our collaborative agreement with Dr. Randy Fishman from the Condensed Matter Division of Oak Ridge National Laboratory, Juana worked summers in Oak Ridge, where she was actively involved in the study of spin diftusion in ferromagnetic setriconductors, a project she continued after moving to $\mathrm{U}$. of Cincinnati in the spring of 2004 as a postdoctoral Estociate of professor Mark Jarrell. In the fall of 2004, Dr. Moreno accepted a position as man assistant professor at University of North Dakota.

$\overline{-}^{-}$C. Pascu Moca visited our group for a six month period starting in the fall of 2004. Our focus has been spin transport in diaordered systems with spin-orbit (Rashba) interaction.

- Dr. Moca is an associate professor at the University of Oradea, Romania.

$\vdots$.

\section{Collaboration with the Oak Ridge National Labora- $\approx$ tory}

One of the biggest benefits provided by this grant was the opportunity to closely interact with scientists from Oak Ridge National Laboratory. To maximize the impact of the collaboration, I decided early on that my research associates should be a direct part of it by offering them the possibility of meeting and working with some of the best condensed matter scientists. Thus, in each of the summers of 2001 and 2002, Dr. Juana Moreno spent two months working with Dr. Randy Fishman in the Condensed Matter Bivision in Oak Ridge. During this time, Juana got involved in a larger group, that also includes Prof. Mark Jarrell, that studies spin dynanica in ferromagnetic semiconductors. 